\title{
Scanning tunneling microscopy investigations of hydrogen plasma-induced electron scattering centers on single-walled carbon nanotubes
}

\author{
G. Buchs, ${ }^{\text {a) }}$ P. Ruffieux, P. Gröning, and O. Gröning \\ Empa, Swiss Federal Laboratories for Materials Testing and Research, nanotech@surfaces, \\ Feuerwerkerstrasse 39, CH-3602 Thun, Switzerland
}

(Received 8 September 2006; accepted 2 December 2006; published online 3 January 2007)

\begin{abstract}
The authors report on the generation of localized defects on single-walled carbon nanotubes by means of a hydrogen electron cyclotron resonance plasma. The defects have been investigated using scanning tunneling microscopy (STM) and show an apparent topographic height in the STM of $1-3 \AA$. In the vicinity of defects, characteristic superstructures could be observed and the patterns could be simulated using a simple model based on large momentum scattering of the valence electrons. The combination of low structural damage and high electronic activity opens the possibility to tune the electronic transport properties using such defects. (C) 2007 American Institute of Physics. [DOI: 10.1063/1.2428594]
\end{abstract}

Since about a decade single-walled carbon nanotubes (SWCNTs) prove their great potential as active electronic material in such devices as field effect transistors, ${ }^{1,2}$ single electron transistors (SETs), ${ }^{3,4}$ or sensor devices. ${ }^{2}$ Low temperature scanning tunneling microscopy (STM) and spectroscopy as well as low temperature transport measurements have been used extensively to characterize the intrinsic electronic properties of SWCNT, so that in combination with theoretical works a detailed understanding of these intrinsic properties could be established. ${ }^{3}$ It has been evidenced, however, that apart from the intrinsic properties the operation of SWCNT devices can be strongly influenced by local defects. For example, it could be shown for metallic SWCNT that gate voltage dependent fluctuations in the tube resistance originated from resonant electron backscattering ${ }^{5,6}$ by intrinsic defects. ${ }^{7}$ More recently, a scanning gate microscopy study investigating the dependence of the two-terminal transconductance of a SWCNT device on the position of the gate electrode revealed a one-to-one correspondence of the spots of highest transconductance with the position of defects. ${ }^{8}$ In both examples cited here the defects were intrinsic and their exact nature could not be resolved. Nevertheless, these findings clearly indicate that SWCNT electronic devices can be strongly influenced and in future may be controlled by introduction of specific defects. Defects may also be important to create tunneling barriers on metallic SWCNT in order to define a quantum dot (QD). In experimentally realized SET, the pair of tunneling barriers that define the QD is usually created by the high resistance metallic contacts to the SWCNT. That defects can take over the role of the contact has been proven by the observation of confined electronic states between the end of a metallic SWCNT and an intrinsic defect of unknown nature. ${ }^{9}$ This observation indicates the possibility to electronically decouple parts of a SWCNT with tunneling barriers formed by atomic defects. This would offer the possibility to achieve QD on SWCNT with large level spacing due to electron confinement.

Today, however, our understanding on the influence of local atomic defects such as Stone-Wales, vacancies, or

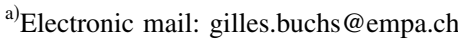

chemisorption on the electronic properties of SWCNT is mostly based on theoretical works ${ }^{6,10}$ and only very few experimental data exist. ${ }^{11,12}$ In this letter we report on the creation of local defects on SWCNT by means of low energy hydrogen ions created by an electron cyclotron resonance (ECR) plasma. The defects are then characterized by means of low temperature scanning tunneling microscopy (LT-STM).

The experiments are carried out in a commercial LTSTM system (Omicron) operating at a base pressure below $10^{-10}$ mbar. The measurements presented in this work were all performed at $77 \mathrm{~K}$. Topography images were recorded in constant-current mode with the set point voltage $V_{s}$ applied to the sample. Gold-on-glass slides ${ }^{13}$ were used as substrates. In order to obtain $\mathrm{Au}$ (111) monoatomic terraces, the gold surface was first relaxed under gentle ex situ hydrogen flame annealing followed by several in situ sputtering and annealing $\left(410^{\circ} \mathrm{C}\right)$ cycles in the STM ulta high vacuum preparation chamber. The SWCNT from Rice University were grown by the high pressure $\mathrm{CO}$ disproportionation process (HiPCo) and purified. ${ }^{14}$ The raw material was sonicated for 2-3 $\mathrm{h}$ in 1,2-dichloroethane in order to break the bundles, and then a droplet of the SWCNT suspension was deposited ex situ on the well prepared gold substrate. After introducing the sample in the LT-STM system, residues from the solvent of the suspension were removed by annealing the sample to $390{ }^{\circ} \mathrm{C}$ for a few minutes. A $2.45 \mathrm{GHz}$ ECR plasma source is fitted to the preparation chamber of the LT-STM and consists of a cylindrical stainless steel resonator cavity of $3 \mathrm{~cm}$ long and $4 \mathrm{~cm}$ in diameter, with a central cylindrical molybdenum antenna of $1.75 \mathrm{~cm}$ in diameter. The magnetic field used to realize the ECR condition in the chamber is created by six permanent magnets placed ex situ around the resonator cavity. Typical plasma treatments were performed at a hydrogen pressure of about $8 \times 10^{-2}$ mbar and a microwave power of $60 \mathrm{~W}$. The sample is positioned $6 \mathrm{~cm}$ under the plasma region and can be moved horizontally by maximally $7 \mathrm{~cm}$ away from the antenna axis. The ion energy distribution measured with an electrostatic analyzer showed that $85 \%$ of the hydrogen ions are comprised in a narrow energy region around $1 \mathrm{eV}$ and the maximum detected energy is 


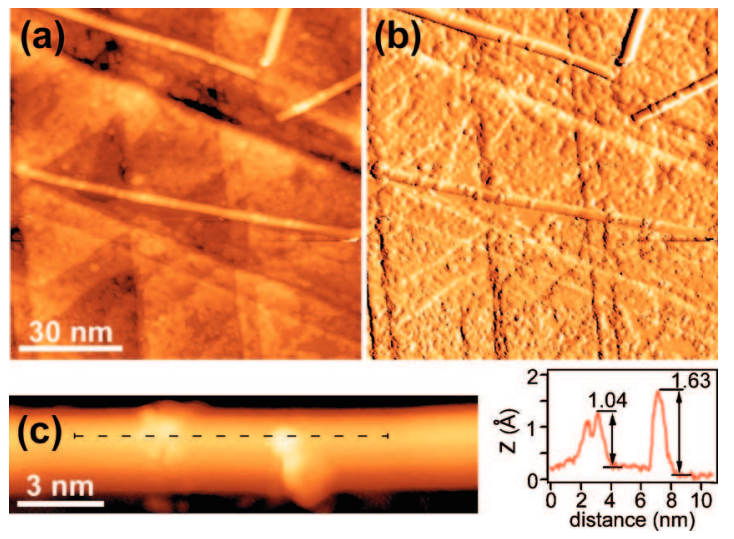

FIG. 1. (Color) (a) Overview STM topographic image of hydrogen plasma irradiated SWCNTs on Au (111), $\Delta z=20 \AA$. Feedback parameters are $V_{s}$ $=1 \mathrm{~V}$ and $I_{s}=0.1 \mathrm{nA}$. (b) STM current image of the same region as in (a), $\Delta I=40 \mathrm{pA}$. (c) STM topographic image and height profile of an individual SWCNT with two defect sites, $\Delta Z=8 \AA, V_{s}=1 \mathrm{~V}$, and $I_{s}=0.1 \mathrm{nA}$.

$\sim 16 \mathrm{eV}$. A typical ion flux with the sample positioned right under the antenna is of the order of $2 \times 10^{13} \mathrm{~s}^{-1} \mathrm{~cm}^{-2} .{ }^{15}$

In order to study the long-range electronic effects of individual plasma-induced defects, we aim at an average defect separation of about $10 \mathrm{~nm}$. To achieve this defect density, we determined the appropriate sample position and irradiation time parameters by test irradiation and STM characterization on highly oriented pyrolitic graphite (HOPG). In comparison with STM images of the SWCNT recorded just before the irradiation process, we observed additional hillocklike features on the treated tubes, where the atomic resolution is lost. The ratio between artificially created and intrinsic defects could be estimated to be larger than 20. Figures 1(a) and 1(b) show, respectively, STM topographic and current error signal overview images of irradiated SWCNT under the conditions mentioned above. The relative height of the observed hillocklike features is in general comprised between 1 and $3 \AA$ and the lateral size varies between 5 and $20 \AA$. A similar relative height was observed in high-energetic argon ion irradiated carbon nanotubes, giving rise essentially to vacancies. ${ }^{10,11}$ The estimated average density of defects along the tubes is about $0.13 \mathrm{~nm}^{-1}$. We showed in an earlier work $^{16,17}$ that exposure of HOPG to an ECR hydrogen plasma as described above mainly resulted in the creation of two types of defects: firstly, chemisorption of hydrogen on the basal plane of graphite and, secondly, carbon vacancies. On HOPG, the formation of adsorption defects was observed with about four times higher occurrence compared to the formation of carbon vacancies. A similar behavior is expected on SWCNT, where possibly more chemisorption sites can be expected. This statement results from the fact that for SWCNT bucky papers exposure to atomic hydrogen (which is of course also present in large quantities in the ECR plasma) is sufficient to create $\mathrm{H}$-chemisorption sites due to the curvature-induced lowering of the adsorption energy barrier. ${ }^{15}$ Theoretical or experimental reports of STM investigations on hydrogen chemisorption at SWCNT are largely lacking in the literature. Figure 1(c) presents a STM topographic image of an individual SWCNT with two defect sites separated by about $4 \mathrm{~nm}$, showing the capability of our method to create defect pairs in view to eventually build intratube QDs. Very often it can be observed that the defect site seems to form a kind of ring around the circumference of Downloaded 30 May 2007 to 152.88.42.154. Redistribution subject
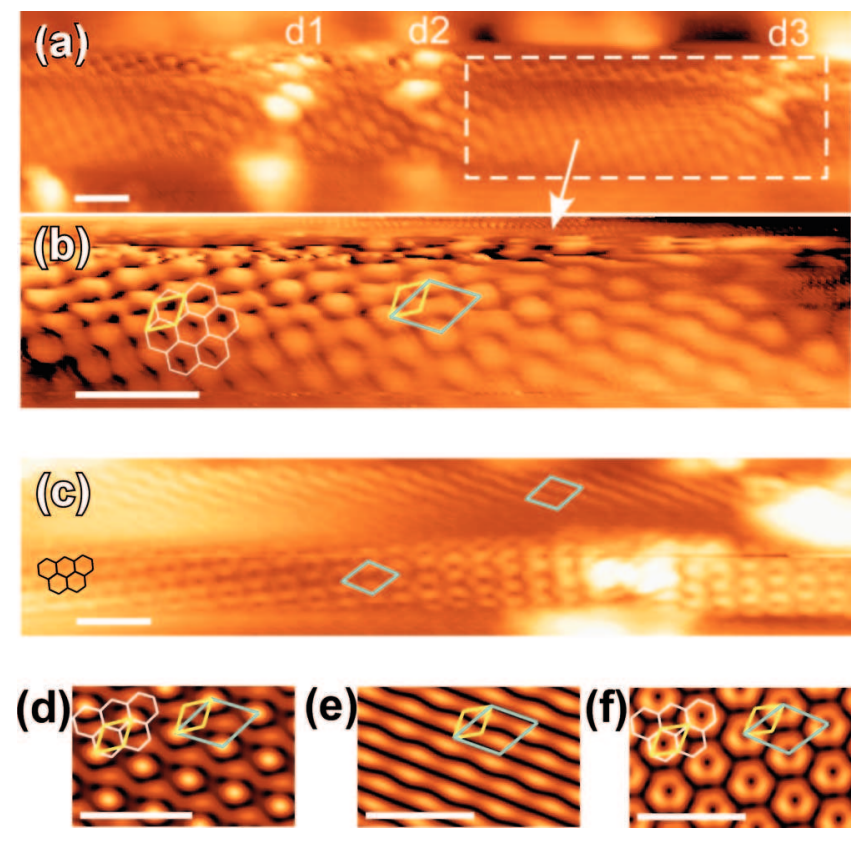

FIG. 2. (Color) (a) STM topography image of a semiconducting tube in a bundle $\left(V_{s}=1 \mathrm{~V}, I_{s}=0.1 \mathrm{nA}, \Delta z=2.4 \AA\right.$, the image is flattened for higher contrast). Three defect sites are indicated by the labels d1-d3. (b) Detail STM image of (a) showing the region between $\mathrm{d} 2$ and $\mathrm{d} 3$, as indicated by the dashed rectangle in (a). Close to $\mathrm{d} 3$ the $(\sqrt{3} \times \sqrt{3}) R 30^{\circ}$ reconstruction of the charge density can be well observed. The $1 \times 1$ and $(\sqrt{3} \times \sqrt{3}) R 30^{\circ}$ unitcells are indicated. On the left hand side the unperturbed honeycomb lattice is evidenced. (c) STM topography image of two metallic SWCNTs showing two different reconstruction patterns near defect sites $\left(V_{s}=1 \mathrm{~V}, I_{s}=0.1 \mathrm{nA}\right.$, and $\Delta z=1.63 \AA$ ) . [(d)-(f)] Calculated spatial maps of $|\psi(\mathbf{r})|^{2}$ according to Eq. (1). Appropriate values of the complex numbers $\phi_{j}$ have been selected to match the experimentally observed patterns in (b) and (c). Scale bars in each image are $1 \mathrm{~nm}$.

the tube. This might be explained by an electronic effect of a defect-induced Friedel-like oscillation resonating around the circumference of the tube.

On HOPG especially the vacancy defects proved to be strong electron scatterers leading to characteristic $(\sqrt{3} \times \sqrt{3}) R 30^{\circ}$ reconstructions in STM images in the vicinity of the defects. $^{16,17}(\sqrt{3} \times \sqrt{3}) R 30^{\circ}$ reconstructions were already observed on SWCNT with intrinsic defects of unknown nature. ${ }^{18}$ Figure 2(a) shows a STM topographic image at $V_{s}=1 \mathrm{~V}$ and $I_{s}=0.1 \mathrm{nA}$ of a tube in a bundle with three defect sites. Spectroscopic $I-V$ curves specified a semiconducting tube. Figure 2(b) displays a zoomed image at $V_{s}=0.63 \mathrm{~V}$ in the region between defects $\mathrm{d} 2$ and $\mathrm{d} 3$, separated by about $6 \mathrm{~nm}$. To compensate the distortion effects due to the curvature of the tubes and the tip shape, the image was contracted in the vertical direction to match the correct angle of $30^{\circ}$ between the armchair and zigzag directions. ${ }^{19}$ It revealed a $(\sqrt{3} \times \sqrt{3}) R 30^{\circ}$ superstructure of the local density of states indicated by the large unit cell. On the left side of the defect $\mathrm{d} 3$, the superstructure contribution decays with a characteristic length of about $5 \mathrm{~nm}$ and the usual graphene unit cell is visible. In Fig. 2(c), we can see two metallic tubes in a bundle, presenting two different $(\sqrt{3} \times \sqrt{3}) R 30^{\circ}$ reconstruction patterns on both sides of defect zones characterized by an increased relative height. On both tubes, the superstructure continuously evolves into the primitive lattice beyond $8 \mathrm{~nm}$.

On HOPG, the different observed $(\sqrt{3} \times \sqrt{3}) R 30^{\circ}$ reconstructions patterns could be reproduced by a linear combinato AlP license or copyright, see http://apl.aip.org/apl/copyright.jsp 
tion of six plane waves having vectors corresponding to the six Fermi vectors of the first Brillouin zone, given by Eq. (1),

$$
\psi(\mathbf{r})=\sum_{j=1}^{6} \phi_{j} \cdot e^{i \mathbf{k}_{j} \cdot \mathbf{r}} .
$$

It could be concluded that the interference patterns originate from large momentum scattering of the electron wave functions at the defects, where the scattering vector of $2 \mathbf{k}_{\mathbf{F}}$ is reduced to $\mathbf{k}_{\mathbf{F}}$ by an Umklapp process. ${ }^{17}$ The expression given by Eq. (1) can also explain the reconstruction patterns on metallic SWCNT because of the band crossing at the Fermi points. In Figs. 2(e) and 2(f), appropriate values of the complex numbers $\phi_{j}$ in Eq. (1) have been selected so that $|\psi(\mathbf{r})|^{2}$ matches the experimentally observed $(\sqrt{3} \times \sqrt{3}) R 30^{\circ}$ reconstruction patterns on the metallic SWCNT in Fig. 1(c). For semiconducting tubes such as in Fig. 2(b), theoretical works report the presence of hillocklike features and superstructures near vacancies due to the contribution of the band edges. ${ }^{10}$ Even tough the model described by Eq. (1) must be modified to take into account the nature of the wave functions present at the band edges, it was possible to simulate the reconstruction pattern observed on the semiconducting SWCNT in Fig. 2(b). All these observations lead to the conclusion that the plasma-induced defects act as in the case of HOPG as strong, large momentum electron scatterers on the SWCNT.

In summary, we showed that hydrogen plasma-induced defects give rise to strong local perturbation of the electronic structure of SWCNT revealed by STM images with hillocklike features on defect sites between 1 and $3 \AA$ in height and a lateral size between 5 and $20 \AA$. Measurements near the defect sites showed $(\sqrt{3} \times \sqrt{3}) R 30^{\circ}$ superstructures with ranges of about 5 and $8 \mathrm{~nm}$ for semiconducting and metallic SWCNT, respectively, indicating large momentum electron backscattering on the plasma-induced defects, as observed on HOPG surface irradiated with the same hydrogen ECR plasma. Low dose hydrogen plasma-induced defects then showed their capability to create strong scatterers on SWCNT and their potential to decouple sections of SWCNT with the important advantage to leave their overall structure intact. The combination of low structural damage and high electronic activity of these defects opens the possibility to tune the electronic transport properties using such defects. Further deeper investigations in the spectroscopy are needed to understand the exact nature of the defects and also their effects on the electronic properties of the SWCNT.

The authors like to acknowledge financial support by the Swiss National Center of Competence in Research MANEP.

${ }^{1}$ S. J. Tans, A. R. M. Verschueren, and C. Dekker, Nature (London) 393, 49 (1998).

${ }^{2}$ P. Avouris, Acc. Chem. Res. 35, 1026 (2002).

${ }^{3}$ C. Dekker, Phys. Today 52, 22 (1999).

${ }^{4}$ H. W. Ch. Postma, T. Teepen, Z. Yao, M. Grifoni, and C. Dekker, Science 293, 76 (2001).

${ }^{5}$ M. Ouyang, J.-L. Huang, and C. M. Lieber, Phys. Rev. Lett. 88, 066804 (2002).

${ }^{6}$ H. J. Choi, J. Ihm, S. G. Louie, and M. L. Cohen, Phys. Rev. Lett. 84, 2917 (2000).

${ }^{7}$ M. Bockrath, W. Liang, D. Bozovic, J. H. Hafner, C. M. Lieber, M. Tinkham, and H. Park, Science 291, 283 (2001).

${ }^{8}$ Y. Fan, B. R. Goldsmith, and P. G. Collins, Nat. Mater. 4, 906 (2005).

${ }^{9}$ T. Maltezopoulos, A. Kubetzka, M. Morgenstern, R. Wiesendanger, S. G. Lemay, and C. Dekker, Appl. Phys. Lett. 83, 1011 (2003).

${ }^{10}$ A. V. Krasheninnikov, K. Nordlund, M. Sirviö, E. Salonen, and J. Keinonen, Phys. Rev. B 63, 245405 (2001).

${ }^{11}$ Z. Osváth, G. Vértesy, L. Tapasztó, F. Wéber, Z. E. Horváth, J. Gyulai, and L. P. Biró, Phys. Rev. B 72, 045429 (2005).

${ }^{12}$ A. Hassanien, M. Tokumoto, P. Umek, D. Vrbanič, M. Mozetič, D. Mihailović, P. Venturini, and S. Pejovnik, Nanotechnology 16, 278 (2005).

${ }^{13}$ www.arrandee.com

${ }^{14}$ I. W. Chiang, B. E. Brinson, A. Y. Huang, P. A. Willis, M. J. Bronikowski, J. L. Margrave, R. E. Smalley, and R. H. Hauge, J. Phys. Chem. B 105, 8297 (2001).

${ }^{15}$ P. Ruffieux, O. Gröning, M. Bielmann, P. Mauron, L. Schlapbach, and P. Gröning, Phys. Rev. B 66, 245416 (2002).

${ }^{16}$ P. Ruffieux, O. Gröning, P. Schwaller, L. Schlapbach, and P. Gröning, Phys. Rev. Lett. 84, 4910 (2000).

${ }^{17}$ P. Ruffieux, M. Melle-Franco, O. Gröning, M. Bielmann, F. Zerbetto, and P. Gröning, Phys. Rev. B 71, 153403 (2005).

${ }^{18}$ W. Clauss, D. J. Bergeron, M. Freitag, C. L. Kane, E. J. Mele, and A. T. Johnson, Europhys. Lett. 47, 601 (1999).

${ }^{19}$ L. C. Venema, V. Meunier, Ph. Lambin, and C. Dekker, Phys. Rev. B 61, 2991 (2000). 